\title{
Development and Application of a New Steady-Hand Manipulator for Retinal Surgery
}

\author{
Ben Mitchell, John Koo, M.D., Iulian Iordachita, Peter Kazanzides, Ankur Kapoor, \\ James Handa, M.D., Gregory Hager, and Russell Taylor
}

\begin{abstract}
This paper describes the development and initial testing of a new and optimized version of a steady-hand manipulator for retinal microsurgery. In the steady-hand paradigm, the surgeon and the robot share control of a tool attached to the robot through a force sensor. The robot controller senses forces exerted by the operator on the tool and uses this information in various control modes to provide smooth, tremor-free, precise positional control and force scaling. The steady-hand manipulator reported here has been specifically designed with the unique constraints of retinal microsurgery in mind. In particular, the system makes use of a compact wrist design that places the bulk of the robot away from the operating field. The resulting system has high efficacy, flexibility and ergonomics while meeting the accuracy and safety requirements of microsurgery. We have now tested this robot on a biological model system and we report a protocol for reliably cannulating $\sim 80 \mu \mathrm{m}$ OD veins (the size of veins in the human retina) using the system.
\end{abstract}

\section{INTRODUCTION}

Many areas of clinical practice involve the manipulation of extremely small, delicate structures. Such structures occur in several organ systems, but are prevalent in the eye, ear, nervous system, and elements of the circulatory system. Within the eye, the manipulation of vitreoretinal structures is particularly difficult given their relative delicacy, inability to regenerate if injured, the surgical inaccessibility, and suboptimal instrumentation to visualize these structures (see Fig. 1). As an initial application for our robot system, we chose retinal vein cannulation, which is the insertion of a needle into a vein on the surface of the retina as a path for drug delivery. This is a procedure which is not safe to perform clinically today, but would have great clinical utility if it could be done reliably. As such, it is a good demonstration of the system's potential.

\section{A. Limitations of current Retinal Microsurgical practice}

During vitreoretinal surgery, the surgeon must visualize the pathology on a micron scale and manually correct the pathology using direct contact, free hand techniques. Procedures occur within the confines of a very small space that is surrounded on all sides by vital structures.

At present, the conventional vitreoretinal system uses an operating microscope to visualize surgical instruments that are placed in three sclerotomy incisions (holes in the sclera

This work was partially funded by the National Science Foundation (NSF) under Engineering Research Center grant EEC9731748, and by the Johns Hopkins University internal funds.

Materials and knowledge relevant to micro-injection were generously provided by the Halpern lab of the Carnegie Institution Department of Embryology.

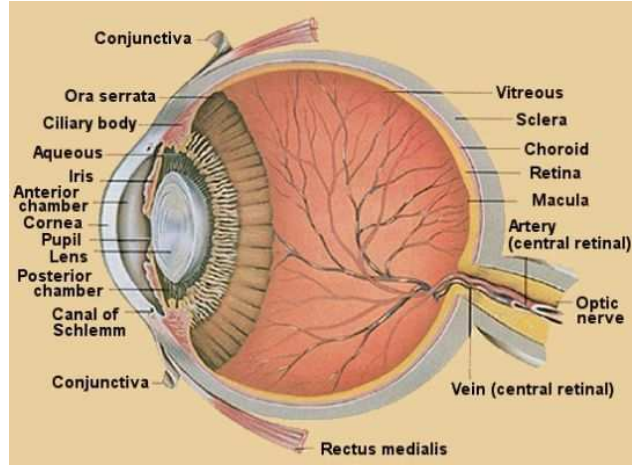

Fig. 1. The anatomy of the human eye

20-25 gauge in diameter). A number of vitreoretinal procedures can then be attempted, including vein cannulation. However, to date retinal vein cannulation does not have a high enough success rate to be used clinically. This is primarily due to the technical difficulty of the procedure caused by visualization limitations, excessive tremor, and insufficient fine motor control. Physiological tremor, which contributes to long operative times and which is exacerbated by fatigue, is a severe limiting factor in microsurgery [14]. Manual dexterity, precision, and perception are particularly important during tasks where the ability to position instruments with great accuracy often correlates directly with the results of the procedure [14], [20]. In a recent study, the root mean square (RMS) amplitude of the tremor of an ophthalmic surgeon under surgical conditions was measured to be $182 \mu \mathrm{m}$ [16]. While it may be possible to briefly position an instrument at a specified target with great accuracy, maintaining the position for extended periods of time becomes increasingly difficult due to physical, visual and mental fatigue [3]. The robotic assistant system described in this paper is designed to help surgeons overcome these difficulties.

There is extensive literature reporting robotic systems for surgery (e.g., [19]), including commercially deployed systems (e.g., [6]). A number of researchers have proposed master-slave microsurgical systems (e.g., [10]), including some systems for the eye ([5]). With the exception of exploratory work by Hunter et al.[9], most of this work has focused on direct improvement of a surgeon's ability to manipulate tissue remotely or at a very fine scale, rather than exploiting the ability of the computer to assist the surgeon more broadly.

In contrast, the JHU Steady-Hand Robot (SHR) [12], [18] was designed to cooperatively share control of a surgical tool 
with the surgeon while meeting the performance, accuracy, and safety requirements of microsurgery. The absolute operational positioning precision is approximately 5 microns. However, this first prototype had serious limitations that prevented it from becoming a clinically useful system. In particular, the parts of the mechanism nearest the patient were bulky and ergonomically inconvenient for the surgeon. This paper describes our second prototype, which is designed to overcome these limitations, and our preliminary success using the robot to cannulate $\sim 80 \mu \mathrm{m}$ OD veins in a biological model system.

\section{Mechanical System Design}

The design of our second robot prototype began with an analysis of the necessary degrees of freedom (DOF), options for obtaining a remote center of motion (RCM), and establishment of specifications for mechanical parameters such as range of motion, precision, and maximum velocity. These are discussed in the following sections.

\section{A. Degrees of Freedom (DOF) Analysis}

We critically analyzed the necessary DOF in tool positioning for eye surgery. There are three phases in surgical tool motion: approach phase (A), insertion phase (I), and retinal surgery phase $(\mathrm{R})$. In the approach phase, the surgeon requires at least $3 \mathrm{DOF}(\mathrm{X}, \mathrm{Y}$, and $\mathrm{Z}$ ) to bring the tool to the entry point on the eye surface (sclerotomy incision). Although these 3 DOF could be realized by many combinations of rotary and translational axes, we chose a Cartesian design (XYZ stage). In the insertion phase, the surgeon requires 3 DOF (one translation plus two rotations). For the retinal surgery phase, four DOF are required: three rotations and one translation (Fig. 2).

The three rotations are local DOF and are necessary for tool orientation. In our evaluation of the manual retinal surgery procedures, we learned that the tool tip positioning accuracy is not very sensitive to the tool spin. We therefore decided to drive only the tool tilt and roll motions, leaving the spin motion for manual manipulation. The insertion could be a local DOF or generated by combining the general DOF (first three DOF). We chose the latter solution. The advantage is that we eliminate a DOF, which allows a more compact design, while the disadvantage is that we require coordinated motion of three axes to produce the insertion motion. This makes it more challenging to obtain high accuracy and, as discussed in the next section, is not consistent with the philosophy of a remote center of motion (RCM) kinematic design. Thus, the new robot has only 5 DOF: 3 translations (general DOF) and two rotations (local DOF). By eliminating two local DOF (tool insertion and spin), we gain the ability to create a thin tool holder and reduce the interaction between the robot and microscope work space.

As for the range of motion, taking into the account the size of the eye, its location on the face, and the insertion point position on the eye, we estimated that for the tool motion close to and inside of the eye, we need a work space around $50 \times 50 \times 50 \mathrm{~mm}$, and for the tool orientation, around

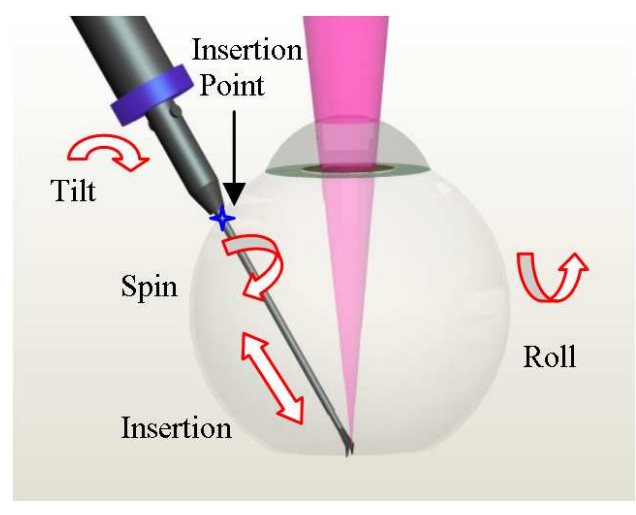

Fig. 2. Setup in retinal surgery phase.

$\pm 30^{\circ}$ about each axis of rotation. Taking into account the necessary space in the approach phase, we set the final range of translation motions at $\pm 50 \mathrm{~mm}$. Because of variability in the configuration of the human face, it could be necessary to increase the rotating angles and/or to set different relative positions of the robot with respect to the patient.

\section{B. Real RCM Point versus Virtual RCM Point}

The retinal surgery phase requires tool motions to be constrained by an insertion point (i.e., the sclerotomy). As shown in Fig. 2, the allowable motions are the three rotations about the insertion point and the translation of the tool through the insertion point. This implies a remote center of motion (RCM), where the three rotation axes intersect at the insertion point. An RCM robot achieves this by mechanical design [17]. Furthermore, many RCM designs include a final actuator to provide the tool insertion (this can also be thought of as a way to translate the RCM point along the tool axis). A real (mechanical) RCM design provides several advantages for surgical applications, such as increased safety due to the minimal number of actuators that must be powered to achieve each task motion. It is also possible to achieve an RCM point by using software to coordinate the robot joints (i.e., a virtual $\mathrm{RCM}$ ), but this can reduce the accuracy and safety of the tool motions.

This discussion of a real (mechanically constrained) versus virtual RCM point is relevant to the design of the tilt mechanism. This mechanism must be precise, assure the necessary range of motion, be compact, and have a remote center of motion that coincides with the insertion point. We analyzed many solutions for the robot wrist by analogy with welding robots. Finally, we considered three mechanisms: a parallel six-bar mechanism with a geometrically imposed RCM [15], [17], a parallel six-bar mechanism with offset (also with RCM) [8], and a slider-crank mechanism (not an RCM). While a real RCM has the advantages listed above, for this system we placed more value on a compact design with high stiffness and accuracy. For this reason we chose to implement the slider-crank mechanism, with a virtual RCM.

\section{Mechanical System Specifications}

In establishing the specifications for the robot mechanical system, we considered its interaction with patient anatomical 
TABLE I

ROBOT PERFORMANCE SPECIFICATIONS FOR APPROACH PHASE (A), INSERTION PHASE (I), AND RETINAL SURGERY PHASE (R) MOTIONS.

\begin{tabular}{|l|l|l|}
\hline Robot Specification & Units & Value \\
\hline Roll/tilt motion & degrees & \pm 30 \\
\hline XYZ motion & $\mathrm{mm}$ & \pm 50 \\
\hline Roll/tilt precision & radians & 0.00005 \\
\hline XYZ precision & $\mu \mathrm{m}$ & 2 \\
\hline Net precision at retina & $\mu \mathrm{m}$ & 5 \\
\hline Cartesian tip speed - phase A & $\mathrm{mm} / \mathrm{s}$ & 10 \\
\hline Cartesion tip speed - phase I & $\mathrm{mm} / \mathrm{s}$ & 5 \\
\hline Cartesian tip speed - phase R & $\mathrm{mm} / \mathrm{s}$ & $<1$ \\
\hline
\end{tabular}

structures, surgeon workspace, and imaging system. Other important factors were the patient safety in correlation with surgery accuracy. The preliminary system specifications are given in Table I.

\section{Mechanical System Components}

The robot mechanical system consists of three major parts (Fig. 3): the XYZ system, the roll mechanism, and the tilt mechanism. The XYZ system controls the global motions of the surgical tool. The roll mechanism, consisting of a rotating table, was tilted at $-15^{\circ}$ from the horizontal direction to allow better access of the surgical tool to the eye depression of the patient face. This roll mechanism configuration is appropriate for the actual tilt mechanism type and for a robot located on the same side of the face as the targeted eye. If the robot location is on the other side of the face, it is necessary to avoid collision with the patient's nose, which could be accomplished by increasing the tilt angle or by tilting the robot using a passive arm. For the current prototype, the roll mechanism allows a $360^{\circ}$ range of rotation for the tool. We chose this motion range so that we could test the robot on many different simulated surgical procedures.

The tilt mechanism (slider-crank) is attached to the roll mechanism through a long tubular arm. In this way, nearly the entire robot is away from the surgery area. Also, this configuration allows for easier separation of the non-sterilized robot from the sterilized surgical area. The translating joint of the tilt mechanism is realized by a rotary motor and a micrometer screw without backlash. To eliminate the translating joint backlash, the slider was realized from two parts that make contact on an oblique surface. The two parts are pushed against each other by a nut through a wave spring.

A 6-DOF force sensor is rigidly attached to the crank (the last element of the tilt mechanism). A tool holder is located between the force sensor and the surgical tool. This is a very important part of the robot: it must be sterilizable, it must be attached to the force sensor through an emergency release mechanism, it must allow the spinning rotation of the tool, and it must provide a precise and easy attachment for the tool. For the current prototype, we implemented only the last two functions. Because of the variability in size and shape of the surgical tools used in retinal surgery, it may be necessary to develop custom adapters for each tool type. At that time it will be possible to make a decision regarding the emergency release mechanism.

\section{SySTEM IMPLEMENTATION}

\section{A. Mechanical Implementation}

The manipulator itself consists of four modular subassemblies: 1) An off-the-shelf XYZ translation assembly; 2) A roll mechanism; 3) A tilt mechanism; 4) Specialized instruments held in the tool holder.

The XYZ translation assembly is formed by mounting a single axis Z-stage orthogonal to a dual axis $\mathrm{X}-\mathrm{Y}$ table (NEAT: LM-400 and NEAT: XYR-6060, respectively, from New England Affiliated Technologies of Lawrence, MA). Each axis consists of a crossed-roller way mounted table actuated by an encoded DC servo motor driven leadscrew. The travel along each axis is $100 \mathrm{~mm}$, and the positioning resolution is $<2.5 \mu \mathrm{m}$ ( $1 \mu \mathrm{m}$ encoder resolution).

For the roll mechanism, we employed a rotary table model B5990TS from Velmex, Inc. Bloomfield, NY, motorized with a DC motor RE 25, 10 Watt connected through a planetary gearhead GP 26 B (14:1 reduction), and encoded with a Digital MR Encoder (512 counts per turn) from Maxon Motor AG. The range of motion is $\pm 180^{\circ}$ with a repeatability of 1 arc-second.

The tilt mechanism (Fig. 4) consists of a custom-made slider-crank mechanism attached to the rotary table through a carbon fiber tube. The slider mechanism, included in the tube, utilizes a high precision lead screw (80 TPI, OD $1 / 4$ inch, sensitivity $1 \mu \mathrm{m} / \mathrm{inch}$ ) from Newport Corporation, Irvine CA, motorized with a DC Maxon motor RE 16, 4.5 Watt connected through a planetary gearhead GP 16 A (19:1 reduction), and encoded with a Digital MR Encoder (512 counts per turn). The crank motion range is $\pm 30^{\circ}$ relative to the vertical tool position. Attached to the crank there is a small commercially available force/torque sensor (Model: NANO-17 SI 12/0.12, ATI Industrial Automation, $\mathrm{NC}$ ), which has force resolutions of $0.0125 \mathrm{~N}$ along the $\mathrm{X}, \mathrm{Y}$ axes, $0.025 \mathrm{~N}$ in the $\mathrm{Z}$ direction, and torque resolutions of $0.0625 \mathrm{~N}-\mathrm{mm}$ about the $\mathrm{X}, \mathrm{Y}$ and $\mathrm{Z}$ axes. Force ranges of $\pm 22.5 \mathrm{~N}$ in the $\mathrm{Z}$-axis and $\pm 12.5 \mathrm{~N}$ in the $\mathrm{X}-\mathrm{Y}$ axes can be measured.

The tool holder facilitates the attachment of a variety of surgical instruments, such as forceps, needle holder, and scissors, that are required during microsurgical procedures. The current prototype has a tool attachment that consists of a manually actuated rigid coupling with a tapered sleeve mounted inside a tubular shaft.

The new prototype of our new steady-hand robot is complete (Fig. 7). The control system has been implemented and the whole system was functionally tested. Also, 3D visualization software was added to the system; the current system allows simultaneous visualization through the stereo oculars on the microscope and on an external stereo-video monitor.

\section{B. Software Implementation}

Over the past few years, our group has developed several admittance control guidance methods, often called virtual fixtures [2], [1], [7]. In general, these methods operate as 


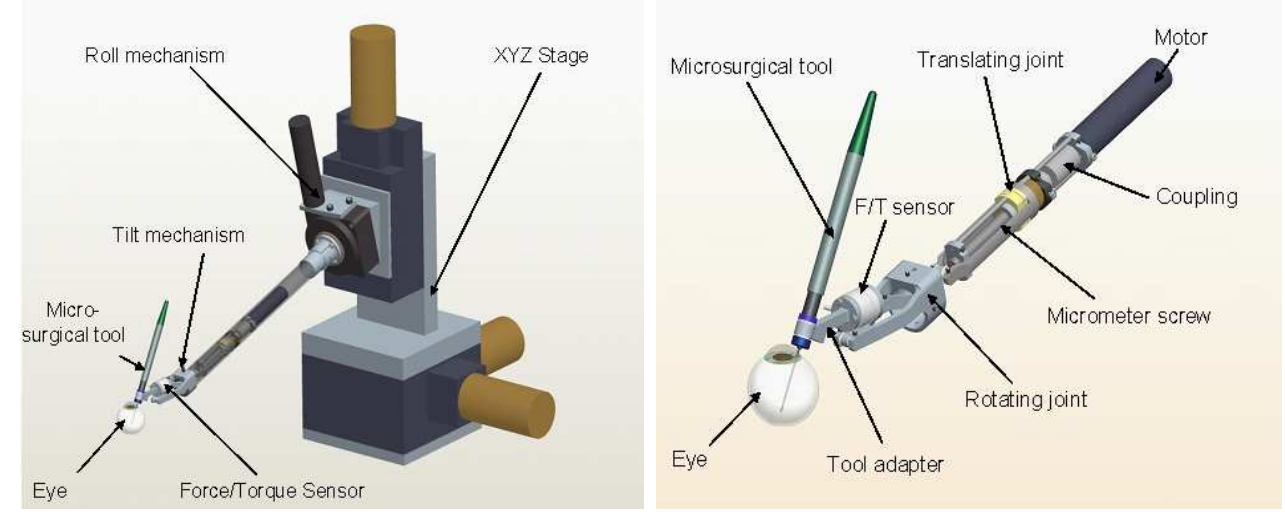

Fig. 3. Robot mechanical system (rendering of CAD model): general view (left) and tilt mechanism (right).

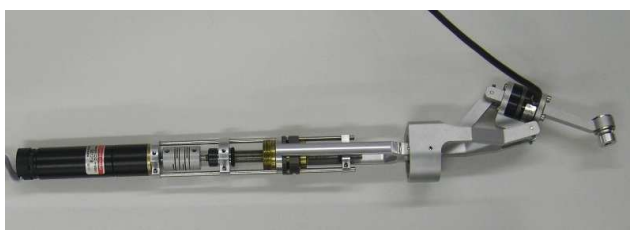

Fig. 4. Robot tilt mechanism

follows: given sensed handle forces and torques $f \in \Re(6)$ exerted by the operator, a tool velocity screw is computed as

$$
v=G f
$$

The matrix $G \in \Re(6 \times 6)$ determines the relative gains of the sensed forces, and thus permits shaping of the motion response to force inputs.

To achieve the virtual RCM point, we use a constrained optimization formulation based on [11]. In particular, we optimize the following cost function:

$$
\left\|J_{h}(q) \Delta q-G f\right\|
$$

subject to the constraint:

$$
\left\|P_{c l}+J_{c l}(q) \Delta q-P_{o}\right\| \leq \epsilon
$$

Here, $P_{c l}$ is a point on the robot tool that is closest to the virtual RCM point, $P_{o} . J_{h}(q)$ and $J_{c l}(q)$ are the manipulator Jacobians resolved at the handle and at $P_{c l}$, respectively, and $q$ is the vector of joint positions (see Fig. 5). The output of this optimization is the desired joint velocity vector, $\Delta q$, which becomes the input to an inner velocity control loop.

Our current software platform is a PC running the Windows operating system. The higher-level admittance control loop is executed on the PC (in soft real time), whereas the inner velocity control loop is performed by a dedicated motion control board (PCX/DSP, Motion Engineering Inc.).

\section{EXPERIMENTAL METHODS AND REsults}

\section{A. Chicken Embryos as Eye Phantoms}

We validated the design of our robot system by testing the ability of a user to successfully complete the vein cannulation task. Constructing a mechanical eye phantom is problematic due to the difficulty of simulating a pressurized, fluid filled tube with the tensile properties of a vein. Using

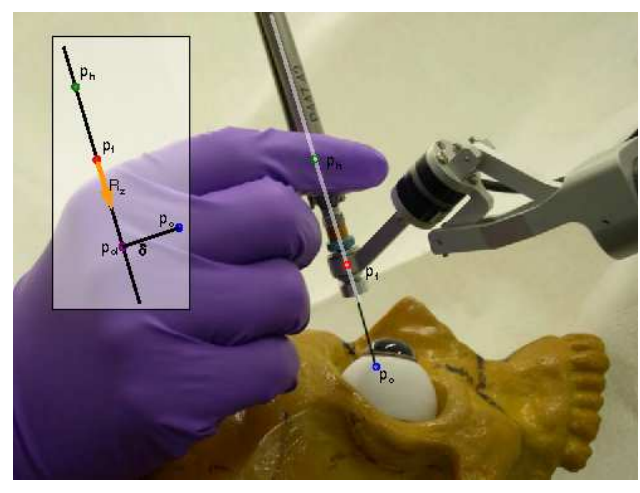

Fig. 5. Virtual RCM mechanism

a biological system for our initial tests makes our success or failure much more clinically relevant. As reported in [13], the chorioallantoic membrane (CAM) of a 12 day old chicken embryo (Fig. 6-B) provides a good model for testing equipment and procedures for retinal surgery. In particular, at around 12 days, the secondary veins on the surface of the CAM are of a size that closely matches the size of the veins on the surface of the human retina. Based on this finding, we used CAM veins as a model to work out and demonstrate a protocol for reliably cannulating veins the size of those on the human retina. The CAM contains a large number of veins which cover a wide range of sizes; after taking measurements to calibrate the microscope, we attempted to cannulate veins between $80 \mu \mathrm{m}$ and $120 \mu \mathrm{m}$. Veins on the human retina range from $40 \mu \mathrm{m}$ to $350 \mu \mathrm{m}[13]$.

\section{B. Microinjection}

The vein cannulation task discussed in this paper is not one that is in use clinically; however, a number of similar micro-injection tasks are routine procedures in fields such as transgenics, embryology, and developmental biology. In each of these fields, pulled glass micro-pipettes are used to do micro-injection. Procedures are done freehand if the target is large enough, or with the help of a micro-manipulator if the target is very small. The success rates of these procedures are typically quite low, which is why they are not used clinically. For more information on micro-pipettes and micro-injection, see [4]. 
It is worth noting that commercially available micromanipulators are not suitable for a surgical setting for several reasons. First, these devices generally have only the three Cartesian degrees of freedom, since they are intended primarily for use in settings with few spatial constraints. They also have a very small workspace and limited dynamics. Our robot has none of these disadvantages, and also has the advantages of a multi-modal controller which allows for dynamic scaling of the tool velocity relative to the measured force on the tool.

\section{Materials and Methods}

The following is a procedure that we have developed for reliable cannulation of $\sim 80 \mu \mathrm{m}$ veins and injection of a visible marker. First, a tool designed to hold a glass micropipette was attached to the Steady-Hand Robot tool-holder. A small flexible tube was run from the back of this tool to a 5-ml syringe, and the syringe was filled with mineral oil that had been put through a $0.2 \mu \mathrm{m}$ filter. The syringe was then clamped into an infusion pump, and the oil was pushed through the system until all air had been removed.

A pulled glass micro-pipette was visualized under an operating microscope, and a razor blade was used to break off the tip at approximately a $45^{\circ}$ angle. This created a hollow tip with an outer diameter of approximately 10-20 $\mu \mathrm{m}$. Since the break was done by hand, each micro-pipette tip was slightly different, but this variation did not prove a significant hindrance in the cannulation procedure.

The micro-pipette was then clamped into the tool, and the infusion pump was used to push the oil down to the tip of the pipette.

Once the injection system was prepared, a 12-14 day old chicken embryo was prepared as described in [13] to expose the CAM, and placed under the microscope (Figs. 6, 7). A neutral phosphate buffer solution (PBS) was used to keep the top of the CAM moist; this kept the CAM from stiffening and improved visualization of the CAM through the microscope.

The steady-hand robot was then used to position the micropipette near an $\sim 80 \mu \mathrm{m}$ vein in the CAM (Fig. 8-A), and the infusion pump was used to put enough pressure on the syringe to ensure a slow but steady flow of oil out of the micro-pipette tip. The steady-hand robot was then used to cannulate the vein.

The actual cannulation proved more difficult than expected, primarily due to the structural properties of the CAM in which the veins were embedded. This membrane is highly elastic so that small amounts of pressure on a vein embedded in it causes significant local membrane deformation. This, combined with the toughness of the vein walls, means that simply touching the pipette tip to the vein is not enough. In order to penetrate the vein, enough pressure must be applied to the vein to deform the membrane so that the elasticity of the membrane exerts enough counter-pressure on the pipette tip to puncture the vein wall.

Exerting this pressure straight down is ineffective, as the rebound when the vein wall is punctured is generally enough to cause the pipette to puncture the far wall of the vein as

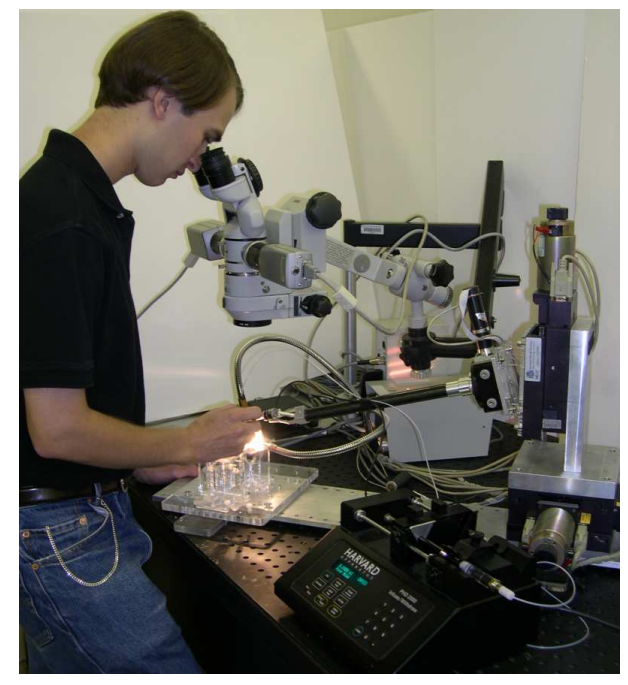

Fig. 7. The new steady-hand manipulator for retinal surgery during a CAM vein cannulation.

well. This results in a torn vein and attendant hemorrhaging. Ideally, we would exert pressure parallel to the vein, because this would mean that excess force would drive the pipette farther into the vein rather than out the far side, but this is not physically possible. The technique which worked best involved first pushing down at a moderately steep angle, pushing along the axis of the vessel, and then lifting up and pushing axially while simultaneously rotating the tool shaft down toward the vein axis using a sort of hooking motion. Done properly, this results in the pipette tip pushing on one side of a small loop of vein, with the direction of force taking the tip farther along the vein, rather than out the other side. See Fig. 8 for a sequence of images taken during a successful cannulation. A short video of the same attempt may be found along with the electronic form of the conference proceedings, or may be downloaded at http: //www. cs . jhu . edu/CIRL/misc/movies. html.

\section{Results and Discussion}

Success or failure of a cannulation attempt was easily determined by whether the oil was inside or outside the vein. Because of its viscous and immiscible properties, the oil tended to stay in large droplets which were easily seen in the buffer solution. For the same reasons, oil within the vasculature showed up as spherical pockets in which blood cells were noticeably absent. When injecting oil into small enough veins, it was found that the blood pressure was not enough to carry the oil away; this would result in large sections of the vasculature being completely filled with the clear oil (Fig. 8-F).

In our initial trials, it was found that an experienced user of the robot can generally cannulate $\sim 80 \mu \mathrm{m}$ veins in under a minute; larger veins can be cannulated even faster. Once a vein has been successfully cannulated, the robot allows the user to maintain the cannulation with no further effort; the tool can be released completely, and will stay in the vein indefinitely. There is no limit to how much fluid can be injected using the system; longer injections do not result in 

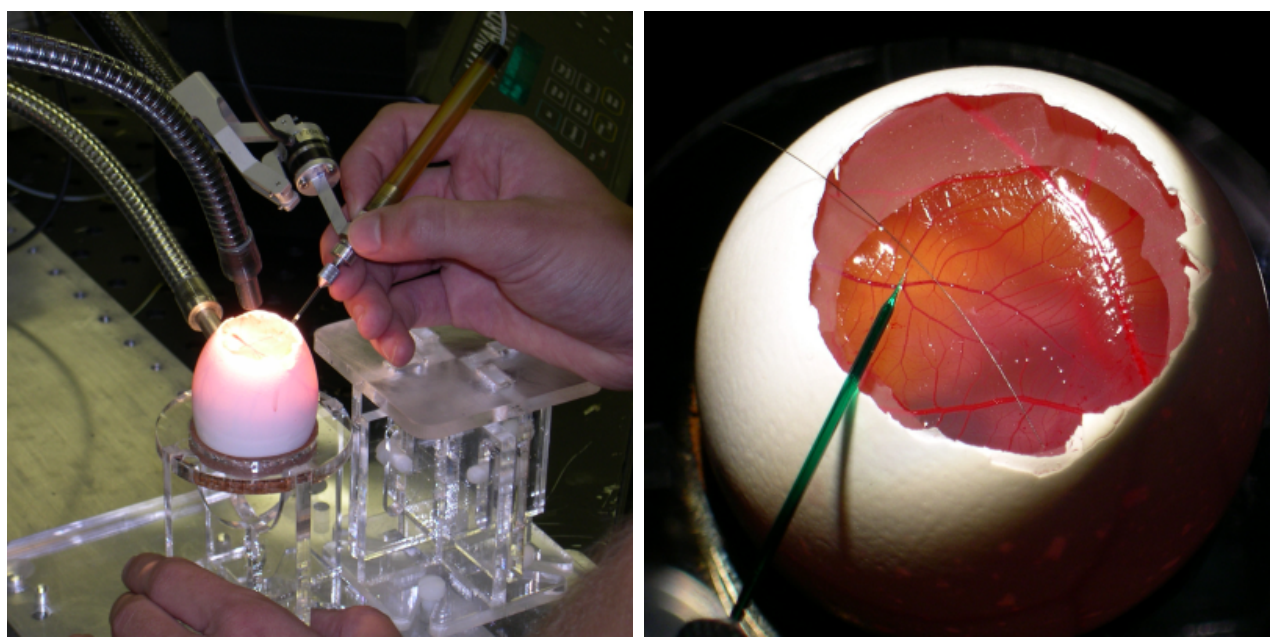

Fig. 6. (A) The tool holder and micro-injection tool during a cannulation. (B) Chicken embryo CAM with a micro-pipette

greater damage to the vein or the surrounding tissue.

While an experienced user was able to cannulate veins of a similar scale freehand (i.e., holding the tool without the help of the robot), there was noticeably more damage to the vein and the surrounding tissue. The tissue surrounding the vein in the CAM is of little anatomical importance, but the same can not be said of the human retina. Longer injections resulted in continued damage to the vein, and the length of time which a cannulation could be maintained freehand was finite. In many instances, a successful cannulation would last only seconds, even for an experienced user with little hand tremor. Even in the best possible scenario, a sustained cannulation for more than about a minute is nearly impossible.

Additionally, the primary limitations on the size of veins which could be cannulated were the visual resolution of the microscope and the outer diameter of the micro-pipette tip. With better micro-pipette manufacturing processes and a higher resolution microscope, it is our belief that the robot could be used to successfully cannulate veins smaller than $80 \mu \mathrm{m}$ without difficulty. The smaller the scale, the greater the proportional damage from hand tremor, and the greater the benefits of the robot's precision.

\section{CONClusions And Future Work}

\section{A. Conclusions}

We have designed and fabricated an advanced and optimized version of a new steady-hand manipulator for retinal surgery. Our approach extends earlier work on cooperative manipulation in microsurgery and focuses on performance augmentation.

In our experiments with CAM veins, we used the new steady-hand robot to successfully cannulate veins down to $\sim 80 \mu \mathrm{m}$ rapidly, reliably, and with minimal damage to the surrounding tissue. Additionally, we developed a protocol for reliably injecting an easily visible marker into the veins, allowing quick, clear visualization of the success or failure of a cannulation attempt. Initial experimentation with this system has shown it to be a reliable and replicable experimental testbed.

\section{B. Future Work}

Having demonstrated that the steady-hand robot system is capable of performing the cannulation task, we plan to extend our work in several directions.

First, all testing to date has been done by only a handful of individuals; we are in the process of performing a usability study with a number of ophthalmology residents from the Johns Hopkins Medical Institute to explore the benefits, drawbacks, and learning curve of the robot in a statistically robust way.

Second, while the CAM is a reasonable model system for initial testing, it is not an actual retina. Unlike the chicken CAM, the human retina is in a pressurized environment[21]. It is thought that this will prevent the veins from moving excessively, somewhat negating the need for the insertion technique described above. It is worth noting that the elasticity of the membrane is actually a help rather than a hindrance in free-hand attempts, because small amounts of movement in the pipette tip simply move the vein in the membrane, rather than damaging it. This means that small amounts of hand tremor are less damaging to the vein and the surrounding tissue than they would be in a more rigid system. It also means that once a vein has been cannulated, it is easier to remain within it freehand, since small amounts of tremor are more likely to move the vein with respect to the chicken egg than move the pipette with respect to the vein.

Likewise, although our insertion technique was used with great success on veins in the CAM, it is not immediately applicable to retinal surgery. This is because the workspace around the chicken egg is unconstrained, and the tool can be maneuvered freely through all 6 degrees of freedom. As noted previously, in retinal surgery the tool must be constrained to move through an RCM due to the necessity of working through the sclerotomy incisions.

To address these issues, we plan to perform tests on real retinas in rabbits, a much better model system. This will facilitate the improvement and refinement of the robot, which will become more rigid, more accurate, and better 


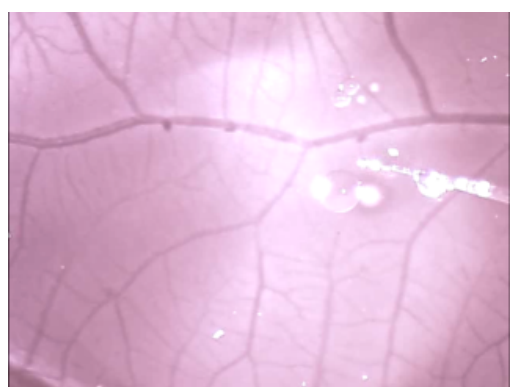

(A)

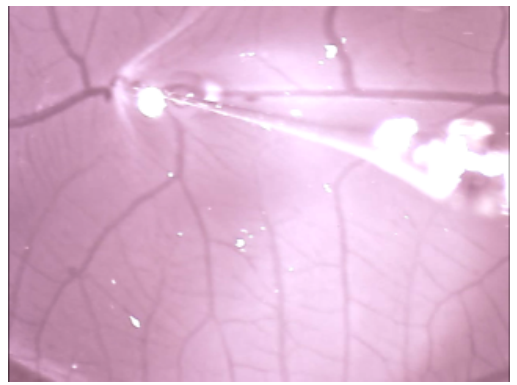

(D)

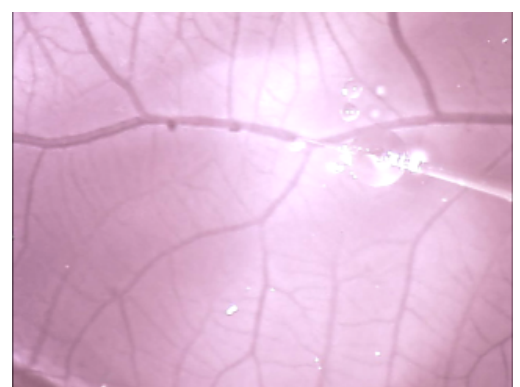

(B)

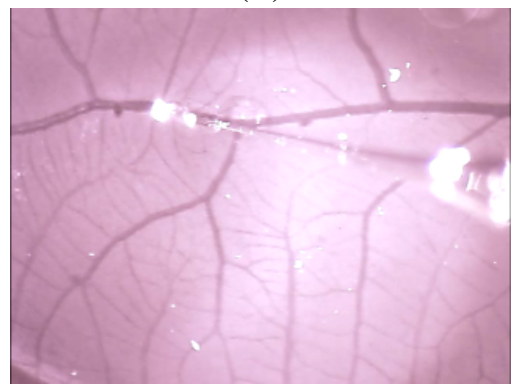

(E)

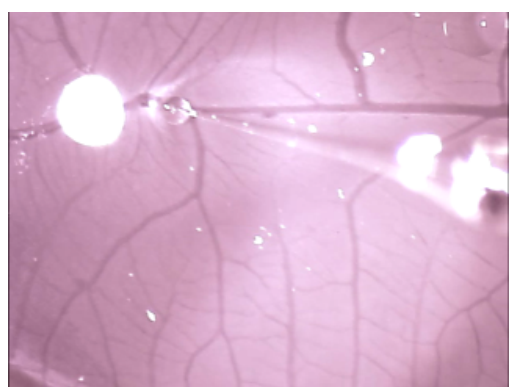

(C)

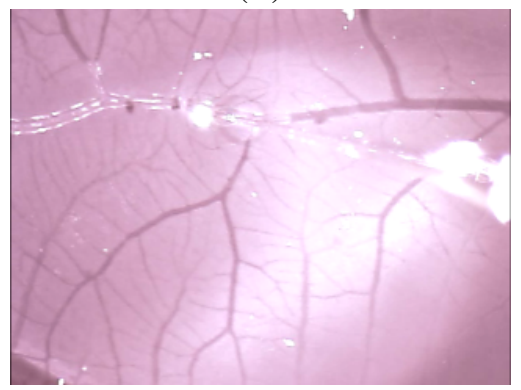

(F)

Fig. 8. Successive images of a cannulation taken through a microscope: (A) tip is positioned near target vein. (B) tip is touching target vein. (C) tip is pushed against target vein. (D) tip is pulled up using hooking motion. (E) tip is pulled back to allow vein to un-distort. (F) tip has not been moved; vein is filled with clear marker.

suited to the ergonomics of micro-surgery. We also plan to build a second steady-hand robot to allow for two-handed manipulations and procedures.

Finally, extensive long-term work is being done to improve the feature set of the microsurgical workstation. This work includes stereo tracking of the tool and the retina for localization, velocity-based virtual fixtures using this localization, and intra-operative use of pre-operative imaging in the form of information fusion and visual overlays on a stereo-video display. Work is also being done on making the robot OR-compatible by introducing sterilizable and/or disposable components.

\section{REFERENCES}

[1] J. J. Abbott and A. M. Okamura. Virtual fixture architectures for telemanipulations. In IEEE Intl. Conf. on Robotics and Automation, pages 2798-2806, Taipei, Taiwan, Sep 2003.

[2] A. Bettini, P. Marayong, S. Lang, A. Okamura, and G. Hager. Vision assisted control for manipulation using virtual fixtures. IEEE Trans. on Robotics and Automation, 20(6):953-966, Dec 2004.

[3] K.R. Boff and J.E. Lincoln. Engineering data compendium: Human perception and performance. H.G. Anderson Aerospace Medical Research Laboratory, Ohio, 1988.

[4] K.T. Brown and D.G. Flaming. Advanced Micropipette Techniques for Cell Physiology, volume 9 of IBRO Handbook Series: Methods in the Neurosciences. Wiley-Interscience, 1986.

[5] S. Charles, R.E. Williams, and B. Hamel. Design of a surgeon-machine interface for teleoperated microsurgery. Proc. Of the Annual Intl Conf. of the IEEE Engineering in Medicine and Biology Society, pages 883884, 1989.

[6] C.K. Chui, H.T. Hguyen, Y. Wang, R. Mullick, R. Raghavan, and J. Anderson. Potential field and anatomy vasculature for real time computation in davinci. 1996.

[7] D. Kragic, P. Marayong, M. Li, A.M. Okamura, and G.D. Hager. Human-Machine Collaborative Systems for Microsurgical Applications. The International Journal of Robotics Research, 24(9):731-741, 2005.

[8] G.J. Hamlin and A.C. Sanderson. Tetrobot: a modular approach to reconfigurable parallel robotics. London, Kluwer Academic, 1995.
[9] I.W. Hunter, D. Doukoglou, S.R. Lafontaine, G. Charette, L.A. Jones, M.A. Sagar, G.D. Mallison, and P.J. Hunter. A teleoperated microsurgical robot and associated virtual environment for eye surgery. Presence, 2:256-280, 1993.

[10] K. Ikuta, K. Yamamoto, and K. Sasaki. Development of remote microsurgery robot and new surgical procedure for deep and narrow space. IEEE Conference on Robotics and Automation, Taiwan, pages 1103-1108, 2003.

[11] A. Kapoor, M. Li, and R.H. Taylor. Constrained control for surgical assistant robots. In IEEE Intl. Conf. on Robotics and Automation, pages 231-236, Orlando, FL, May 2006.

[12] R. Kumar, G.D. Hager, A. Barnes, P. Jensen, L.L. Whitcomb, and R.H. Taylor. An augmentation system for fine manipulation. in proceeding of medical image computing and computer assisted intervention. Lecture Notes in Computer Science, 1935 Springer-Verlag:956-965, 2000.

[13] T. Leng, J.M. Miller, K.V. Bilbao, D.V. Palanker, P. Huie, and M.S Blumenkranz. The chick chorioallantoic membrane as a model tissue for surgical retinal research and simulation. Retina, 24(3):427-434, June 2004.

[14] M Patkin. Ergonomics applied to the practice of microsurgery. Aust NZ J Surg, 47:320-329, 1977.

[15] M. Rosheim. Robot Wrist Actuators. Wiley, 1989.

[16] S. Sinch and C. Riviere. Physiological tremor amplitude during retinal microsurgery. Proc. 28th IEEE Northeast Bioeng. Conf, Philadelphia, pages 171-172, 2002.

[17] R.H. Taylor, J. Fund, D.D. Grossman, J.P. Karidis, and D.A. LaRose. Remote centor-of-motion robot for surgery. US Patent 5,397,323, Mar. 14, 1995.

[18] R.H. Taylor, P. Jensen, L.L. Whitcomb, A. Barnes, R. Kumar, D. Stoianovici, P. Gupta, Z. Wang, E. de Juan, and L. Kavoussi. Steady-hand robotic system for microsurgical augmentation. The International Journal of Robotics Research, 18(12):1201-1210, 1999.

[19] R.H. Taylor and D. Stoianovici. Medical robotics in computerintegrated surgery. IEEE Transactions on Robotics and Automation, 19:765-781, 2003.

[20] H.P. Weinrib. Rotational technique and microsurgery. Microsurgery, 5:207-212, 1984.

[21] G. Wollensak, E. Spoerl, C. Wirbelaur, and D.T. Pham. Influence of indocyanini green staining on the biomechanical strength of porcine internal limiting membrane. Ophthalmologica, 218(4):278-282, JulAug 2004. 